\title{
Regularity of n-generalized Schützenberger product of monoids
}

\author{
Esra KIRMIZI ÇETÍNALP* \\ Karamanoğlu Mehmetbey University, Kamil Özdă̆ Science Faculty, \\ Department of Mathematics, Yunus Emre Campus, Karaman.. \\ Geliş Tarihi (Received Date): 25.03.2021 \\ Kabul Tarihi (Accepted Date): 05.07.2021
}

\begin{abstract}
In this work, regularity of $n$-generalized Schützenberger product of monoids from the point of Group Theory is studied. Here, it is determined necessary and sufficient conditions of the $n$-generalized Schützenberger product $A_{1} \diamond A_{2} \diamond \cdots \diamond A_{n}$ to be regular while all $A_{i}(1 \leq i \leq n)$ are monoids. Also, by considering all $A_{i}(1 \leq i \leq n)$ to be groups, it is given another result for the regularity of this product.
\end{abstract}

Keywords: Schützenberger product, monoid, regularity.

\section{Monoidlerin n-genelleştirilmiş Schützenberger çarpımının regülerliği}

Öz

$\mathrm{Bu}$ çalışmada, monoidlerin $n$-genelleştirilmiş Schützenberger çarpımın regülerliği Grup Teori açısından incelenmiştir. Burada, bütün $A_{i}(1 \leq i \leq n)$ 'ler monoid iken $A_{1} \diamond A_{2} \diamond \cdots \diamond A_{n} \quad n$-genelleştirilmiş Schützenberger çarpımın regüler olabilmesi için gerekli ve yeterli koşul elde edilmiştir. Ayrıca, bütün $A_{i}(1 \leq i \leq n)$ 'leri grup düşünerek bu çarpımın regülerliği için bir diğer sonuç verilmiştir.

Anahtar kelimeler: Schützenberger çarpım, monoid, regülerlik.

*Esra KIRMIZI ÇETINALP, esrakirmizi@kmu.edu.tr, http://orcid.org/0000-0002-3754-8622 


\section{Introduction and preliminaries}

Recent developments in group and semigroup theory have raised the question of whether there exists a classification of some algebraic structures according to regularity, $\pi$-inverse, $p$-Cockcroft property. As an answer to the regularity of this algebraic structures, in [9], Skornjakov explained regularity of the wreath product of monoids. After that, in [7], it has been investigated regularity property of semidirect products of monoids. After these works, in [6], the authors determined necessary and sufficient conditions for Schützenberger product of monoids and the new version of the Schützenberger product of monoids to be regular and strongly $\pi$-inverse. Furthermore, in [3], the authors studied the regularity of a new monoid construction under crossed and Schützenberger product to any two monoids. In this study, as the main result of this paper, we give an answer by defining necessary and sufficient conditions of the $n$ generalized Schützenberger product $A_{1} \diamond A_{2} \diamond \cdots \diamond A_{n}$ to be regular where all $A_{i}(1 \leq i \leq n)$ are any monoids.

We recall that a monoid $M$ is called regular if, for every $a \in M$, there exists $b \in M$ such that $a b a=a$ and $b a b=b$ (or, equivalently, for the set of inverses of $a$ in $M$, that is, $a^{-1}=\{b \in B: a b a=a, b a b=b\}, M$ is regular if and only if, for all $a \in M$, the set $a^{-1}$ is not equal to the emptyset.

The Schützenberger product is an operation on monoids that was originally introduced for solving questions in automata theory and to analyze the syntactic properties of the concatenation product in formal language theory. The Schützenberger product was originally defined by Schützenberger (1965) ([8]) for two monoids, and extended by Straubing (1981) ([10]) for any number of monoids. In [5], the authors obtained a presentation for Schützenberger product of two monoids and gave the normal form structure of the elements of this product.

Let $M_{1}$ and $M_{2}$ be monoids presented by $\left\langle X_{1} \mid R_{1}\right\rangle$ and $\left\langle X_{2} \mid R_{2}\right\rangle$, respectively. For $P \subseteq M_{1} \times M_{2}, a \in M_{1}, b \in M_{2}$, we define

$$
a P=\{(a c, d) \mid(c, d) \in P\}, P b=\{(c, d b) \mid(c, d) \in P\} .
$$

The Schützenberger product of $M_{1}$ and $M_{2}$, denoted by $M_{1} \diamond M_{2}$, is the set

$$
M_{1} \times \mathrm{P}\left(M_{1} \times M_{2}\right) \times M_{2}
$$

with multiplication $\left(a_{1}, P_{1}, b_{1}\right)\left(a_{2}, P_{2}, b_{2}\right)=\left(a_{1} a_{2}, P_{1} b_{2} \cup a_{1} P_{2}, b_{1} b_{2}\right)$, where $\mathrm{P}\left(M_{1} \times M_{2}\right)$ is the power set obtained from the product sets $M_{1}$ and $M_{2}$. The Schützenberger product of $M_{1}$ and $M_{2}$ is presented by

$$
\begin{gathered}
\wp_{M_{1} \diamond M_{2}}=\langle Z| R_{1}, R_{2}, z_{w_{1}, w_{2}}^{2}=z_{w_{1}, w_{2}}, z_{w_{1}, w_{2}} z_{w_{1}^{\prime}, w_{2}}=z_{w_{1}^{\prime}, w_{2}} z_{w_{1}, w_{2}}, \\
\left.x_{1} z_{w_{1}, w_{2}}=z_{x_{1} w_{1}, w_{2}} x_{1}, z_{w_{1}, w_{2}} x_{2}=x_{2} z_{w_{1}, w_{2} x_{2}}, x_{1} x_{2}=x_{2} x_{1}\right\rangle,
\end{gathered}
$$

where $x_{i} \in X_{i}, w_{i}, w_{i}^{\prime} \in M_{i}(i \in\{1,2\})$ and $Z=X_{1} \cup X_{2} \cup\left\{z_{w_{1}, w_{2}} \mid w_{1} \in M_{1}, w_{2} \in M_{2}\right\}$ (see [5]). 
In [4], the authors gave presentations of the Schützenberger product of $n$ groups $G_{1}, \cdots, G_{n}$, given a monoid presentation $\left\langle X_{i} \mid R_{i}\right\rangle$ of each group $G_{i}$, by using matrix theory. In [2], the authors studied a new $n$-generalized monoid construction of Schützenberger product from view of Combinatorial Group Theory and found a presentation of this generalized product.

Let $A_{1}, A_{2}, \cdots, A_{n-1}$ and $A_{n}$ be monoids. For $P_{i, i+1} \subseteq A_{i} \times A_{i+1}(1 \leq i \leq n-1)$, and $a_{i} \in A_{i}(1 \leq i \leq n)$, we define

$$
\begin{aligned}
& a_{i} P_{i, i+1}=\left\{\left(a_{i} x_{i}, x_{i+1}\right) ; \quad\left(x_{i}, x_{i+1}\right) \in P_{i, i+1}\right\}, \\
& P_{i, i+1} a_{i+1}=\left\{\left(x_{i}, x_{i+1} a_{i+1}\right) ; \quad\left(x_{i}, x_{i+1}\right) \in P_{i, i+1}\right\} .
\end{aligned}
$$

$n$-generalized Schützenberger product of monoids $A_{1}, A_{2}, \cdots A_{n-1}$ and $A_{n}$, denoted by $A_{1} \diamond A_{2} \diamond \cdots \diamond A_{n}$, is the set $A_{1} \times \mathrm{P}\left(A_{1} \times A_{2}\right) \times A_{2} \times \mathrm{P}\left(A_{2} \times A_{3}\right) \times A_{3} \times \cdots \times \mathrm{P}\left(A_{n-1} \times A_{n}\right) \times A_{n}$ with the multiplication

$$
\begin{aligned}
& \left(a_{1}, P_{1,2}, a_{2}, P_{2,3}, a_{3}, \cdots, P_{n-1, n}, a_{n}\right)\left(a_{1}^{\prime}, P_{1,2}^{\prime}, a_{2}^{\prime}, P_{2,3}^{\prime}, a_{3}^{\prime}, \cdots, P_{n-1, n}^{\prime}, a_{n}^{\prime}\right) \\
& =\left(a_{1} a_{1}^{\prime}, a_{1} P_{1,2}^{\prime} \cup P_{1,2} a_{2}^{\prime}, a_{2} a_{2}^{\prime}, a_{2} P_{2,3}^{\prime} \cup P_{2,3} a_{3}^{\prime}, \cdots, a_{n-1} a_{n-1}^{\prime}, a_{n-1} P_{n-1, n}^{\prime} \cup P_{n-1, n} a_{n}^{\prime}, a_{n} a_{n}^{\prime}\right) .
\end{aligned}
$$

For all $a_{i} \in A_{i}$ and $P_{i, i+1} \in \mathrm{P}\left(A_{i} \times A_{i+1}\right), n$-generalized Schützenberger product $A_{1} \diamond A_{2} \diamond \cdots \diamond A_{n}$ defines a monoid with the identity $\left(1_{A_{1}}, \varnothing, 1_{A_{2}}, \cdots, \varnothing, 1_{A_{n}}\right)[2]$.

We finally note that the reader is referred to [1,2] and [4] for a detailed survey on $n$ generalized Schützenberger product.

\section{Regularity of n-generalized Schützenberger product}

In this section, we proved by two different proofs for the regularity of $n$-generalized Schützenberger product of monoids $A_{1}, A_{2}, \cdots, A_{n-1}$ and $A_{n}$. Firstly, we gave necessary and sufficient conditions for $n$-generalized Schützenberger product $A_{1} \diamond A_{2} \diamond \cdots \diamond A_{n}$ to be regular while all $A_{i}(1 \leq i \leq n)$ are arbitrary monoids. Then, by considering all $A_{i}(1 \leq i \leq n)$ to be groups, we give another result for the regularity of this product.

Theorem 2.1 Let $A_{1}, A_{2}, \cdots, A_{n-1}$ and $A_{n}$ be any monoids. Then $n$-generalized Schützenberger product $A_{1} \diamond A_{2} \diamond \ldots \diamond A_{n}$ is regular if and only if,

(i) $\forall A_{i}(1 \leq i \leq n)$ are regular,

(ii) for $\left(a_{1}, P_{1,2}, a_{2}, P_{2,3}, a_{3}, \cdots, P_{n-1, n}, a_{n}\right) \in A_{1} \diamond A_{2} \diamond \cdots \diamond A_{n}$, either 


$$
P_{i, i+1}=a_{i} Q_{i, i+1} a_{i+1}=\bigcup_{\left(x_{i}, x_{i+1}\right) \in Q_{i, i+1}}\left\{\left(a_{i} x_{i}, x_{i+1} a_{i+1}\right)\right\}(1 \leq i \leq n-1),
$$

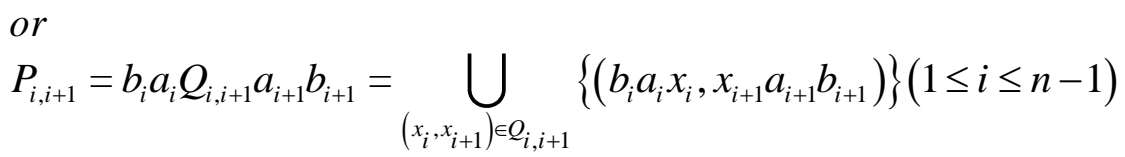

where $P_{i, i+1} \subseteq A_{i} \times A_{i+1}(1 \leq i \leq n-1)$ and $b_{i} \in a_{i}^{-1}(1 \leq i \leq n)$.

Proof. Let us suppose that $A_{1} \diamond A_{2} \diamond \cdots \diamond A_{n}$ is regular. Thus for $\left(a_{1}, \varnothing, a_{2}, \varnothing, a_{3}, \cdots, \varnothing, a_{n}\right) \in A_{1} \diamond A_{2} \diamond \cdots \diamond A_{n}$, there exists $\left(b_{1}, Q_{1,2}, b_{2}, Q_{2,3}, b_{3}, \cdots, Q_{n-1, n}, b_{n}\right)$ such that

$$
\begin{aligned}
\left(a_{1}, \varnothing, a_{2}, \cdots, \varnothing, a_{n}\right) & =\left(a_{1}, \varnothing, a_{2}, \cdots, \varnothing, a_{n}\right)\left(b_{1}, Q_{1,2}, b_{2}, \cdots, Q_{n-1, n}, b_{n}\right)\left(a_{1}, \varnothing, a_{2}, \cdots, \varnothing, a_{n}\right) \\
& =\left(a_{1} b_{1}, a_{1} Q_{1,2}, a_{2} b_{2}, \cdots, a_{n-1} Q_{n-1, n}, a_{n} b_{n}\right)\left(a_{1}, \varnothing, a_{2}, \cdots, \varnothing, a_{n}\right) \\
& =\left(a_{1} b_{1} a_{1}, a_{1} Q_{1,2} a_{2}, a_{2} b_{2} a_{2}, \cdots, a_{n-1} Q_{n-1, n} a_{n}, a_{n} b_{n} a_{n}\right)
\end{aligned}
$$

and

$$
\begin{array}{r}
\left(b_{1}, Q_{1,2}, b_{2}, \cdots, Q_{n-1, n}, b_{n}\right)=\left(b_{1}, Q_{1,2}, b_{2}, \cdots, Q_{n-1, n}, b_{n}\right)\left(a_{1}, \varnothing, a_{2}, \cdots, \varnothing, a_{n}\right)\left(b_{1}, Q_{1,2}, b_{2}, \cdots, Q_{n-1, n}, b_{n}\right) \\
=\left(b_{1} a_{1}, Q_{1,2} a_{2}, \quad b_{2} a_{2}, \cdots, Q_{n-1, n} a_{n}, b_{n} a_{n}\right)\left(b_{1}, Q_{1,2}, b_{2}, \cdots, Q_{n-1, n}, b_{n}\right) \\
=\left(b_{1} a_{1} b_{1}, b_{1} a_{1} Q_{1,2} \cup Q_{1,2} a_{2} b_{2}, b_{2} a_{2} b_{2}, \cdots, b_{n-1} a_{n-1} Q_{n-1, n} \cup Q_{n-1, n} a_{n} b_{n}, b_{n} a_{n} b_{n}\right) .
\end{array}
$$

Therefore, we obtain that $a_{i}=a_{i} b_{i} a_{i}(1 \leq i \leq n)$ and $b_{i}=b_{i} a_{i} b_{i}(1 \leq i \leq n)$. This implies that $(i)$ must hold.

By the assumption on the regularity of $A_{1} \diamond A_{2} \diamond \cdots \diamond A_{n}$ for $\left(a_{1}, P_{1,2}, a_{2}, \cdots, P_{n-1, n}, a_{n}\right) \in A_{1} \diamond A_{2} \diamond \cdots \diamond A_{n}$, we have $\left(b_{1}, Q_{1,2}, b_{2}, \cdots, Q_{n-1, n}, b_{n}\right)$ such that

$\left(a_{1}, P_{1,2}, a_{2}, \cdots, P_{n-1, n}, a_{n}\right)$

$=\left(a_{1}, P_{1,2}, a_{2}, \cdots, P_{n-1, n}, a_{n}\right)\left(b_{1}, Q_{1,2}, b_{2}, \cdots, Q_{n-1, n}, b_{n}\right)\left(a_{1}, P_{1,2}, a_{2}, \cdots, P_{n-1, n}, a_{n}\right)$

$=\left(a_{1} b_{1}, a_{1} Q_{1,2} \cup P_{1,2} b_{2}, a_{2} b_{2}, \ldots, a_{n-1} Q_{n-1, n} \cup P_{n-1, n} b_{n}, a_{n} b_{n}\right)\left(a_{1}, P_{1,2}, a_{2}, \ldots, P_{n-1, n}, a_{n}\right)$

$=\left(a_{1} b_{1} a_{1}, a_{1} b_{1} P_{1,2} \cup a_{1} Q_{1,2} a_{2} \cup P_{1,2} b_{2} a_{2}, a_{2} b_{2} a_{2}, \ldots, a_{n-1} b_{n-1} P_{n-1, n} \cup a_{n-1} Q_{n-1, n} a_{n} \cup P_{n-1, n} b_{n} a_{n}, a_{n} b_{n} a_{n}\right)$

and

$\left(b_{1}, Q_{1,2}, b_{2}, \cdots, Q_{n-1, n}, b_{n}\right)$

$=\left(b_{1}, Q_{1,2}, b_{2}, \cdots, Q_{n-1, n}, b_{n}\right)\left(a_{1}, P_{1,2}, a_{2}, \cdots, P_{n-1, n}, a_{n}\right)\left(b_{1}, Q_{1,2}, b_{2}, \cdots, Q_{n-1, n}, b_{n}\right)$

$=\left(b_{1} a_{1}, b_{1} P_{1,2} \cup Q_{1,2} a_{2}, b_{2} a_{2}, \cdots, b_{n-1} P_{n-1, n} \cup Q_{n-1, n} a_{n}, b_{n} a_{n}\right)\left(b_{1}, Q_{1,2}, b_{2}, \cdots, Q_{n-1, n}, b_{n}\right)$

$=\left(b_{1} a_{1} b_{1}, b_{1} a_{1} Q_{1,2} \cup b_{1} P_{1,2} b_{2} \cup Q_{1,2} a_{2} b_{2}, b_{2} a_{2} b_{2}, \cdots, b_{n-1} a_{n-1} Q_{n-1, n} \cup b_{n-1} P_{n-1, n} b_{n} \cup Q_{n-1, n} a_{n} b_{n}, b_{n} a_{n} b_{n}\right)$

Hence this gives us $a_{i}=a_{i} b_{i} a_{i}, b_{i}=b_{i} a_{i} b_{i}(1 \leq i \leq n)$ and 


$$
\begin{aligned}
& P_{i, i+1}=a_{i} b_{i} P_{i, i+1} \cup a_{i} Q_{i, i+1} a_{i+1} \cup P_{i, i+1} b_{i+1} a_{i+1}(1 \leq i \leq n-1), \\
& Q_{i, i+1}=b_{i} a_{i} Q_{i, i+1} \cup b_{i} P_{i, i+1} b_{i+1} \cup Q_{i, i+1} a_{i+1} b_{i+1}(1 \leq i \leq n-1) .
\end{aligned}
$$

To show the second condition (ii) given in theorem, let us suppose that $P_{i, i+1} \neq a_{i} R_{i, i+1} a_{i+1} \quad$ for some $R_{i, i+1} \subseteq A_{i} \times A_{i+1}(1 \leq i \leq n-1)$. Then there exists $\left(x_{i}, x_{i+1}\right) \in P_{i, i+1}(1 \leq i \leq n-1)$ such that $x_{i} \neq a_{i} x_{i}^{\prime}$ and $x_{i+1} \neq a_{i+1} x_{i+1}^{\prime}$ where $x_{i}^{\prime} \in A_{i}$ and $x_{i+1}^{\prime} \in A_{i+1}$. Thus $P_{i, i+1}$ cannot be equal to $a_{i} b_{i} P_{i, i+1} \cup a_{i} Q_{i, i+1} a_{i+1} \cup P_{i, i+1} b_{i+1} a_{i+1}$ for all $Q_{i, i+1} \subseteq A_{i} \times A_{i+1}(1 \leq i \leq n-1)$. This gives a contradiction with the regularity of $A_{1} \diamond A_{2} \diamond \cdots \diamond A_{n}$.

In fact, if someone choose $P_{i, i+1}=a_{i} R_{i, i+1} a_{i+1}$, then we obtain that

$$
\begin{aligned}
a_{i} b_{i} & P_{i, i+1} \cup a_{i} Q_{i, i+1} a_{i+1} \cup P_{i, i+1} b_{i+1} a_{i+1} \\
& =a_{i} b_{i} a_{i} R_{i, i+1} a_{i+1} \cup a_{i} Q_{i, i+1} a_{i+1} \cup a_{i} R_{i, i+1} \underline{a_{i+1} b_{i+1} a_{i+1}} \\
& =a_{i} R_{i, i+1} a_{i+1} \cup a_{i} Q_{i, i+1} a_{i+1} \cup a_{i} R_{i, i+1} a_{i+1} \quad\left(b y \text { choosing } Q_{i, i+1}=b_{i} a_{i} R_{i, i+1} a_{i+1} b_{i+1}\right) \\
& =a_{i} R_{i, i+1} a_{i+1} \cup \underline{a_{i} b_{i} a_{i} R_{i, i+1}} \underline{a_{i+1} b_{i+1} a_{i+1}} \cup a_{i} R_{i, i+1} a_{i+1} \\
& =a_{i} R_{i, i+1} a_{i+1} \\
& =P_{i, i+1}
\end{aligned}
$$

and

$$
\begin{aligned}
& b_{i} a_{i} Q_{i, i+1} \cup b_{i} P_{i, i+1} b_{i+1} \cup Q_{i, i+1} a_{i+1} b_{i+1} \\
& \left.\quad=b_{i} a_{i} Q_{i, i+1} \cup b_{i} a_{i} R_{i, i+1} a_{i+1} b_{i+1} \cup Q_{i, i+1} a_{i+1} b_{i+1} \quad \text { (by choosing } Q_{i, i+1}=b_{i} a_{i} R_{i, i+1} a_{i+1} b_{i+1}\right) \\
& =b_{i} a_{i} b_{i} a_{i} R_{i, i+1} a_{i+1} b_{i+1} \cup b_{i} a_{i} R_{i, i+1} a_{i+1} b_{i+1} \cup b_{i} a_{i} R_{i, i+1} \underline{a_{i+1} b_{i+1} a_{i+1} b_{i+1}} \\
& =b_{i} a_{i} R_{i, i+1} a_{i+1} b_{i+1} \\
& =Q_{i, i+1} .
\end{aligned}
$$

We say that, by making similar calculation as above for the case $P_{i, i+1}=b_{i} a_{i} R_{i, i+1} a_{i+1} b_{i+1}$ in this theorem, where $R_{i, i+1} \subseteq A_{i} \times A_{i+1}(1 \leq i \leq n-1)$ and $a_{i} \in b_{i}^{-1}(1 \leq i \leq n)$, it is seen that condition (ii) must hold.

Conversely, for the other part of the proof, we take $\left(a_{1}, P_{1,2}, a_{2}, \cdots, P_{n-1, n}, a_{n}\right) \in A_{1} \diamond A_{2} \diamond \cdots \diamond A_{n}$. Thus we definitely have $a_{i} \in A_{i}(1 \leq i \leq n)$ such that $a_{i} \in b_{i}^{-1}$. Now let us consider the union of sets $a_{i} b_{i} P_{i, i+1} \cup a_{i} Q_{i, i+1} a_{i+1} \cup P_{i, i+1} b_{i+1} a_{i+1}$ and $b_{i} a_{i} Q_{i, i+1} \cup b_{i} P_{i, i+1} b_{i+1} \cup Q_{i, i+1} a_{i+1} b_{i+1} \quad(1 \leq i \leq n-1)$. Here, by taking $P_{i, i+1}=a_{i} R_{i, i+1} a_{i+1}$ and by choosing $Q_{i, i+1}=b_{i} a_{i} R_{i, i+1} a_{i+1} b_{i+1} \subseteq A_{i} \times A_{i+1}(1 \leq i \leq n-1)$, then we get 


$$
a_{i} b_{i} P_{i, i+1} \cup a_{i} Q_{i, i+1} a_{i+1} \cup P_{i, i+1} b_{i+1} a_{i+1}=a_{i} R_{i, i+1} a_{i+1}=P_{i, i+1}(1 \leq i \leq n-1)
$$

and

$$
b_{i} a_{i} Q_{i, i+1} \cup b_{i} P_{i, i+1} b_{i+1} \cup Q_{i, i+1} a_{i+1} b_{i+1}=b_{i} a_{i} R_{i, i+1} a_{i+1} b_{i+1}=Q_{i, i+1}(1 \leq i \leq n-1) .
$$

As a result of this, for every $\left(a_{1}, P_{1,2}, a_{2}, \cdots, P_{n-1, n}, a_{n}\right) \in A_{1} \diamond A_{2} \diamond \cdots \diamond A_{n}$, there exists $\left(b_{1}, Q_{1,2}, b_{2}, \cdots, Q_{n-1, n}, b_{n}\right) \in A_{1} \diamond A_{2} \diamond \cdots \diamond A_{n}$ such that

$\left(a_{1}, P_{1,2}, a_{2}, \cdots, P_{n-1, n}, a_{n}\right)\left(b_{1}, Q_{1,2}, b_{2}, \cdots, Q_{n-1, n}, b_{n}\right)\left(a_{1}, P_{1,2}, a_{2}, \cdots, P_{n-1, n}, a_{n}\right)$

$=\left(a_{1} b_{1} a_{1}, a_{1} b_{1} P_{1,2} \cup a_{1} Q_{1,2} a_{2} \cup P_{1,2} b_{2} a_{2}, \cdots, a_{n-1} b_{n-1} P_{n-1, n} \cup a_{n-1} Q_{n-1, n} a_{n} \cup P_{n-1, n} b_{n} a_{n}, a_{n} b_{n} a_{n}\right)$

and

$\left(b_{1}, Q_{1,2}, b_{2}, \cdots, Q_{n-1, n}, b_{n}\right)\left(a_{1}, P_{1,2}, a_{2}, \cdots, P_{n-1, n}, a_{n}\right)\left(b_{1}, Q_{1,2}, b_{2}, \cdots, Q_{n-1, n}, b_{n}\right)$

$=\left(b_{1} a_{1} b_{1}, b_{1} a_{1} Q_{1,2} \cup b_{1} P_{1,2} b_{2} \cup Q_{1,2} a_{2} b_{2}, \cdots, b_{n-1} a_{n-1} Q_{n-1, n} \cup b_{n-1} P_{n-1, n} b_{n} \cup Q_{n-1, n} a_{n} b_{n}, b_{n} a_{n} b_{n}\right)$.

Additionally, by applying the above similar arguments for the case $P_{i, i+1}=b_{i} a_{i} R_{i, i+1} a_{i+1} b_{i+1}$, where $R_{i, i+1} \subseteq A_{i} \times A_{i+1}(1 \leq i \leq n-1)$ and $b_{i} \in a_{i}^{-1}(1 \leq i \leq n)$, the proof of the regularity of $A_{1} \diamond A_{2} \diamond \cdots \diamond A_{n}$ is completed.

Now, we give another result for the regularity of $n$-generalized Schützenberger product $A_{1} \diamond A_{2} \diamond \cdots \diamond A_{n}$ while all $A_{i}(1 \leq i \leq n)$ are groups.

Theorem 2.2 The $n$-generalized Schützenberger product $A_{1} \diamond A_{2} \diamond \cdots \nabla A_{n}$ of $n$ monoids $A_{i}(1 \leq i \leq n)$ is regular if and only if every $A_{i}(1 \leq i \leq n)$ are groups.

Proof. Let $A_{i}(1 \leq i \leq n)$ be monoids and suppose that $A_{1} \diamond A_{2} \diamond \ldots \diamond A_{n}$ is regular. Then for each $a_{i} \in A_{i}$, the element $\left(a_{1},\left\{\left(1_{A_{1}}, 1_{A_{2}}\right)\right\}, a_{2},\left\{\left(1_{A_{2}}, 1_{A_{3}}\right)\right\}, a_{3}, \ldots,\left\{\left(1_{A_{n-1}}, 1_{A_{n}}\right)\right\}, a_{n}\right) \in A_{1} \diamond A_{2} \diamond \cdots \diamond A_{n}$ is regular, that is, there exists $\left(a_{1}^{\prime}, P_{1,2}, a_{2}^{\prime}, P_{2,3}, \cdots, P_{n-1, n}, a_{n}^{\prime}\right) \in A_{1} \diamond A_{2} \diamond \cdots \diamond A_{n}$ such that

$$
\begin{aligned}
&\left(a_{1},\left\{\left(1_{A_{1}}, 1_{A_{2}}\right)\right\}, a_{2},\left\{\left(1_{A_{2}}, 1_{A_{3}}\right)\right\}, a_{3}, \ldots,\left\{\left(1_{A_{n-1}}, 1_{A_{n}}\right)\right\}, a_{n}\right) \\
&=\left(a_{1},\left\{\left(1_{A_{1}}, 1_{A_{2}}\right)\right\}, a_{2},\left\{\left(1_{A_{2}}, 1_{A_{3}}\right)\right\}, \cdots,\left\{\left(1_{A_{n-1}}, 1_{A_{n}}\right)\right\}, a_{n}\right)\left(a_{1}^{\prime}, P_{1,2}, a_{2}^{\prime}, P_{2,3}, \cdots, P_{n-1, n}, a_{n}^{\prime}\right) \\
&\left(a_{1},\left\{\left(1_{A_{1}}, 1_{A_{2}}\right)\right\}, a_{2},\left\{\left(1_{A_{2}}, 1_{A_{3}}\right)\right\}, \cdots,\left\{\left(1_{A_{n-1}}, 1_{A_{n}}\right)\right\}, a_{n}\right) \\
&=\left(a_{1} a_{1}^{\prime}, a_{1} P_{1,2} \cup\left\{\left(1_{A_{1}}, 1_{A_{2}}\right)\right\} a_{2}^{\prime}, a_{2} a_{2}^{\prime}, a_{2} P_{2,3} \cup\left\{\left(1_{A_{2}}, 1_{A_{3}}\right)\right\} a_{3}^{\prime}, \cdots, a_{n-1} P_{n-1, n} \cup\left\{\left(1_{A_{n-1}}, 1_{A_{n}}\right)\right\} a_{n}^{\prime}, a_{n} a_{n}^{\prime}\right) \\
&\left(a_{1},\left\{\left(1_{A_{1}}, 1_{A_{2}}\right)\right\}, a_{2},\left\{\left(1_{A_{2}}, 1_{A_{3}}\right)\right\}, \cdots,\left\{\left(1_{A_{n-1}}, 1_{A_{n}}\right)\right\}, a_{n}\right)
\end{aligned}
$$




$$
\begin{aligned}
& =\left(a_{1} a_{1}^{\prime} a_{1}, a_{1} a_{1}^{\prime}\left\{\left(1_{A_{1}}, 1_{A_{2}}\right)\right\} \cup a_{1} P_{1,2} a_{2} \cup\left\{\left(1_{A_{1}}, 1_{A_{2}}\right)\right\} a_{2}^{\prime} a_{2}, a_{2} a_{2}^{\prime} a_{2}, a_{2} a_{2}^{\prime}\left\{\left(1_{A_{2}}, 1_{A_{3}}\right)\right\} \cup a_{2} P_{2,3} a_{3}\right. \\
& \left.\cup\left\{\left(1_{A_{2}}, 1_{A_{3}}\right)\right\} a_{3}^{\prime} a_{3}, \cdots, a_{n-1} a_{n-1}^{\prime}\left\{\left(1_{A_{n-1}}, 1_{A_{n}}\right)\right\} \cup a_{n-1} P_{n-1, n} a_{n} \cup\left\{\left(1_{A_{n-1}}, 1_{A_{n}}\right)\right\} a_{n}^{\prime} a_{n}, a_{n} a_{n}^{\prime} a_{n}\right) .
\end{aligned}
$$

So,

$$
\begin{aligned}
& \left\{\left(1_{A_{1}}, 1_{A_{2}}\right)\right\}=a_{1} a_{1}^{\prime}\left\{\left(1_{A_{1}}, 1_{A_{2}}\right)\right\} \cup a_{1} P_{1,2} a_{2} \cup\left\{\left(1_{A_{1}}, 1_{A_{2}}\right)\right\} a_{2}^{\prime} a_{2}, \\
& \left\{\left(1_{A_{2}}, 1_{A_{3}}\right)\right\}=a_{2} a_{2}^{\prime}\left\{\left(1_{A_{2}}, 1_{A_{3}}\right)\right\} \cup a_{2} P_{2,3} a_{3} \cup\left\{\left(1_{A_{2}}, 1_{A_{3}}\right)\right\} a_{3}^{\prime} a_{3}, \\
& \quad \ldots \\
& \left\{\left(1_{A_{n-1}}, 1_{A_{n}}\right)\right\}=a_{n-1} a_{n-1}^{\prime}\left\{\left(1_{A_{n-1}}, 1_{A_{n}}\right)\right\} \cup a_{n-1} P_{n-1, n} a_{n} \cup\left\{\left(1_{A_{n-1}}, 1_{A_{n}}\right)\right\} a_{n}^{\prime} a_{n} .
\end{aligned}
$$

Hence, we obtain

$\left(a_{i-1} a_{i-1}^{\prime}, 1_{A_{i}}\right)=\left\{\left(1_{A_{i-1}}, 1_{A_{i}}\right)\right\}(2 \leq i \leq n) \quad$ and $\quad\left(1_{A_{i-1}}, a_{i}^{\prime} a_{i}\right)=\left\{\left(1_{A_{i-1}}, 1_{A_{i}}\right)\right\}(2 \leq i \leq n)$.

Altogether, the monoids $A_{i}(1 \leq i \leq n)$ satisfy

$$
\forall a_{i} \quad \text { and } \exists a_{i}^{\prime}: \quad a_{i} a_{i}^{\prime}=1_{A_{i}} \quad \text { and } \quad a_{i}^{\prime} a_{i}=1_{A_{i}},
$$

all of which statements imply that the monoids $A_{i}(1 \leq i \leq n)$ in assumptions are groups.

Conversely, let us suppose that each $A_{i}(1 \leq i \leq n)$ be groups. Then, the regularity of $n$ generalized Schützenberger product of $n$ groups is obvious.

\section{References}

[1] Çetinalp, E.K., Karpuz, E.G. ve Çevik, A.S., Complete rewriting system for Schützenberger product of $n$ groups, Asian-European Journal of Mathematics, 12 (1), (2019).

[2] Çetinalp, E.K. ve Karpuz, E.G., $n$-Generalized Schützenberger Product of Monoids and Complete Rewriting System, Bulletin Mathematique de la Societe des Sciences Mathematiques de Roumanie, submitted.

[3] Emin, A., Ateş, F., İkikardeş, S. ve Cangül, İ.N., A new monoid construction under crossed products, J. Inequal. Appl., 244 (2013).

[4] Gomes, G.M.S., Sezinando, H. ve Pin, J.E., Presentations of the Schützenberger product of $n$ groups, Communications in Algebra, 34 (4), 1213-1235, (2006).

[5] Howie, J.M. ve Ruskuc, N., Constructions and presentations for monoids, Comm. in Algebra, 22 (15), 6209-6224, (1994).

[6] Karpuz, E.G., Ateş, F. ve Çevik, A.S., Regular and $\pi$-inverse monoids under Schützenberger products, Algebras, Groups and Geometries, 27, 455-471, (2010). 
[7] Nico, W.R., On the regularity of semidirect products, Journal of Algebra, 80, 29-36, (1983).

[8] Schützenberger, M.P., On finite monoids having only trivial subgroups, Information and Control, 8, 190-194, (1965).

[9] Skornjakov, L.A., Regularity of the wreath product of monoids, Semigroup Forum, 18, 83-86, (1979).

[10] Straubing, H., A generalization of the Schützenberger product of finite monoids, Theo. Comp. Sci., 13, 137-150, (1981). 